\title{
Qué está pasando con los valores profesionales en la formación de los médicos. Visión del alumno.
}

\author{
Cheryl Terés Castillo \\ Consejo Estatal de Estudiantes de Medicina (CEEM).
}

\section{DE LA VOCACIÓN A LOS OBJETIVOS}

La Medicina es una ciencia cuyo objeto fundamental es curar el hombre enfermo y, en consecuencia, ha tenido siempre un gran componente humano. Sin embargo, su práctica se ha visto modificada por los avances, tanto científicos como técnicos, de la era actual.

El médico de hoy, ha pasado de ser un profesional liberal a ser un funcionario que, rendido en parte a las exigencias del sistema, parece haber reemplazado la práctica basada en la vocación y los valores médicos por una relación médico-paciente despersonalizada, dando más importancia al sinfín de datos clínicos de los que dispone, al cumplimiento de objetivos, a la realización de tareas administrativas y al mare mágnum burocrático que al proceso de curación en sí mismo.

El cambio, como era de esperar, ha afectado también a la actitud del paciente ante el médico. El paciente dispone más que nunca de una gran cantidad de información, lo que implica mayor exigencia de atención médica y, por lo tanto, facilita la insatisfacción hacia el sistema de salud en general. Desde hace unos años, no es extraordinario para un profesional de la Medicina que un paciente se presente en su consulta con una serie de documentos impresos de Internet que contienen amplia información sobre la patología que adolece. Llegados a este punto al médico no le queda más opción que ponerse a la defensiva y centrarse en evitar una denuncia por parte de los ahora llamados "usuarios" y, por consiguiente, incumplir el código deontológico, que en el artículo 18.2 reza: «El médico no debe indicar exploraciones o tratamientos que no tienen otro fin que su protección. La Medicina defensiva es contraria a la ética médica».

\begin{abstract}
Y esta situación, ¿cómo la viven los estudiantes de Medicina?

La docencia de pregrado en las Facultades de Medicina del Estado Español se divide en dos ciclos.
\end{abstract}

En el primer ciclo se imparten asignaturas que tienen una elevada carga teórica y están divididas en compartimientos estancos, con una nula relación entre si. El estudiante, ya desde primer curso, empieza a estudiar al ser humano por materias separadas si ningún tipo de integración o si esta existe se realiza de una manera insuficiente. Todo esto mayoritariamente enseñado mediante clases magistrales, que resultan poco atractivas y a las cuales muchos estudiantes terminan por no asistir. A esta realidad cabe añadir el agravante de que la gran mayoría de docentes son investigadores de Ciencias Médicas Básicas como biólogos, bioquímicos y licenciados en medicina carentes, todos ellos, de contacto con la práctica clínica. Por último debo hacer referencia a los métodos de evaluación que se resumen principalmente a una nota numérica fruto de un examen exclusivamente teórico en el que se evalúan conocimientos con un nivel de concreción y especificidad que en determinadas ocasiones resulta insólito. Así, los estudiantes mejor valorados son aquellos que tienen mejores resultados académicos, el estudiante estrella es aquel que tiene una mayor capacidad de memorizar o que talvez dedica más tiempo a hacerlo. Por ende, los estudiantes que dedican parte de su tiempo a desarrollar otras actividades y, en consecuencia, no obtienen unos resultados tan buenos en los exámenes, son considerados de menor calidad aunque, talvez, posean mejores habilidades en 
muchos aspectos que aquellos estudiantes con un expediente más alto.

Es normal, pues, que la dimensión humana de la carrera en este primer ciclo, simplemente, no exista. De manera que en lugar de valores como la empatía con el paciente o la humildad, se fomentan la competencia entre compañeros, la autosuficiencia y el trabajo individual, entre otros, valores todos, al parecer, muy bien vistos en la sociedad actual pero que hacen que se pierda la esencia mas humana de la profesión médica y que desde el principio empiezan a distorsionar la visión de la carrera que adquiere el médico en formación.

En el segundo ciclo llega el estudio de las patologías, de nuevo mediante clases magistrales. Y por otra parte la esperada "introducción al medio clínico" que en demasiadas ocasiones se ve frustrada por la realidad de la práctica médica: tutores de prácticas sobrecargados con las tareas asistenciales y de investigación que prefieren -o deben- dejar delado la docencia. De esta forma los estudiantes empiezan a manejarse por el hospital muchas veces sin otra guía que el sentido de supervivencia, buscando a alguien que quiera o pueda enseñarles. Normalmente se trata de residentes que, por su edad $o$ por su reciente experiencia en las Facultades, sienten mayor empatía hacia el estudiante e intentan así saldar el déficit de la estructura docente a nivel clínico.

Con todo esto, el segundo ciclo está, igualmente, desprovisto de cualquier formación humanista en valores y el único modelo a seguir es el de un profesional médico mayoritariamente agotado e insatisfecho que da más importancia al curar que al cómo curar, mediante la ya mencionada "práctica clínica defensiva", que implica el empleo injustificado de pruebas diagnósticas y procedimientos terapéuticos con el propósito de protegerse de potenciales demandas por mala práctica. De ahí que la frustración de los estudiantes de Medicina vaya aumentando y más aún cuando los mismos facultativos, en ocasiones, cuestionan el hecho de que hayas escogido la carrera de Medicina. Todo esto conlleva a que lo que antaño era un bachiller ilusionado con una idealización del médico, la carrera y la profesión y con un gran sentido de la responsabilidad hacia el enfermo y la sociedad, pase a ser un estudiante sobrecargado con los contenidos teóricos y frustrado por el escaso y precario contacto con los pacientes y el medio clínico y, al final, se convierta en un licenciado que accede al sistema de formación MIR con un amplio abanico de conocimientos teóricos pero que se siente inseguro y desprovisto de herramientas para desenvolverse en el medio clínico.

Parece, pues, que la formación en valores es una asignatura pendiente para la Educación Médica en el Estado Español, donde sólo cuatro de las veintiséis Facultades públicas incluyen la Bioética como asignatura obligatoria: la Universitat de Lleida, la Universidad de Sevilla, la Universidad de Zaragoza y la Universidad Complutense de Madrid. Cabe aclarar que algunas otras Facultades enseñan contenidos de Bioética en el marco de otras asignaturas como la Historia de la Medicina o la Psicología Médica o como asignaturas optativas y de libre elección.

Los estudiantes de medicina consideramos que esta situación no está de acuerdo con las necesidades de la sociedad y que la formación en valores debería estar presente de forma transversal a lo largo de toda la carrera constituyendo un marco básico en el que se fundamenten los conocimientos y habilidades impartidos sirviéndose de diferentes disciplinas como la bioética y la psicología médica, entre otras. Pero para conseguirlo hace falta concienciar a profesores y alumnos, adoptar nuevos modelos de enseñanza y evaluación que vayan más allá de los conocimientos técnicos y teóricos. Sería importante que en las Universidades se enseñase la Medicina desde su dimensión más amplia, haciendo hincapié en la importancia que merecen ciertos valores en el contexto de la relación médico-paciente, que es el pilar básico de la praxis médica, permitiendo al estudiante la libertad de escoger después de conocer estos valores y no dejándole en la ignorancia tal como sucede actualmente. Por lo tanto, se debe enfatizar en los valores más relevantes de la práctica médica de manera que los estudiantes tengamos la oportunidad, mediante la reflexión y el autoanálisis, de conocernos mejor a nosotros mismos y a nuestra profesión. Sólo de esta manera pueden formarse profesionales cuya preocupación no sea únicamente establecer un diagnóstico y realizar un tratamiento correcto sino también la atención a la persona que padece una patología.

El cómo se deben impartir estos conocimientos es una cuestión difícil pero no imposible y por eso se debe tener en cuenta la opinión de las sociedades científicas, expertas en educación médica y en deontología. Los estudiantes sabemos que la moral hace parte del individuo y es algo personal, pero sin embargo hay conductas que se pueden adquirir con un entrenamiento adecuado y que pueden facilitar la manera como se desenvuelve el profesional en el 
día a día. De esta manera, desde la Facultad, se debe construir un sistema de salud en el que el paciente como persona sea el centro de la praxis médica. Pero hace falta ir más allá y es por eso que los estudiantes consideramos que en los programas de formación de postgrado, en nuestro caso en los programas de los Médicos Internos Residentes, también deben existir contenidos y deben evaluarse habilidades orientadas a una mejora de la relación no sólo con el paciente sino con el resto del equipo clínico y se debe estimular a los especialistas en formación para que las adquieran facilitándoles el acceso a cursos con este tipo de contenidos. Así se podrían formar profesionales capaces de satisfacer las necesidades de sus pacientes y de devolver a la Medicina el valor social que hasta hace unas décadas había tenido, profesionales que abarquen las patologías desde la tan famosa, pero desafortunadamente poco aplicada, dimensión biopsico-social, capaces de conocer a sus pacientes y de ser algo más que técnicos de diagnóstico.

Los representantes de estudiantes a través del Consejo Estatal de Estudiantes de Medicina (CEEM) hemos decidido tomar acción y hemos creado una comisión de bioética en el seno del Consejo que se ocupe de encontrar soluciones a las diferentes inquietudes que tenemos respecto a la mejora de los programas de formación y de las metodologías docentes en todos estos aspectos y que servirá para canalizar nuestras propuestas. No obstante, somos conscientes de las dificultades que afrontan las Facultades el Estado Español, donde conseguir fondos para mejorar estos aspectos es una tarea ardua y dificultosa y donde resulta más fácil conseguir fondos para estudiar la relación entre ratones y arañas que para mejorar la docencia con miras a formar unos profesionales más competentes en materia de comunicación y relación con los pacientes. Igualmente reconocemos que la situación de los tutores en los hospitales universitarios no es la ideal y reivindicamos que se reconozca su labor docente con méritos de la misma manera que se reconoce la investigación puesto que la docencia de calidad es igualmente importante para la sociedad y requiere la dedicación del tiempo del profesional.

Los conocimientos teóricos tienen fecha de caducidad pero no las competencias relacionadas con el desarrollo personal, la comunicación, la capacidad de resolución y de trabajo en equipo. Valores como la humildad, el sentido del deber, la responsabilidad, el altruismo, la honestidad, la ecuanimidad, la empatía, etc., son importantes en esta profesión y deberían constituir un pilar en la formación de pregrado y, más aún, en la de postgrado. Sólo de esa manera se puede conseguir que la Medicina recupere aquel componente humano que es, en última instancia, el que nos llevó a escoger esta profesión y lo que le hace diferente del resto de profesiones. 